\title{
How to determine the complex refractive index from infrared reflectance spectroscopy?
}

\author{
Thomas Bonnal $^{1} \cdot$ Ali Belarouci $^{2} \cdot$ Regis Orobtchouk $^{3} \cdot$ Elodie Prud'homme $^{4}$ (I) $\cdot$ Solene Tadier ${ }^{4} \cdot$ Genevieve Foray $^{1}$
}

Received: 5 March 2020 / Accepted: 9 November 2020 / Published online: 24 November 2020

(c) Springer Nature Switzerland AG 2020

\begin{abstract}
A new theoretical methodology enabling the determination of the complex refractive index has been developed and explored; it only requires to use a classical set-up to control the incident angle, without the need for a polarizer. As a proof of concept, this new methodology has been successfully applied to investigate the optical properties of amorphous silica in the mid-infrared spectral range and validated by comparison with a standard routine based on polarization state of the light. This work thus makes it possible to determine the complex refractive index of an isotropic material by a reflectance measurement without needing to control the polarization of light. The refractive index can be measured with a relative uncertainty close to $10^{-3}$ over a wide range of wavelengths, as needed for a growing number of optical applications. Therefore, this new technique is cost effective and permits the accurate assessment of the refractive index in the whole infrared range.
\end{abstract}

Keywords Complex refractive index · Fourier transform infrared spectroscopy $\cdot$ Single-angle reflectance $\cdot$ Multi-angles reflectance $\cdot$ Complex optical constants

\section{Introduction}

Recently, light-matter interaction in the infrared range has been used for applications in various areas, such as medicine, environment, high-speed communications or energy. For instance, it is useful to identify tumours $[1,2]$ or to characterize deteriorated binders and materials in ancient wall paintings [3]. It is also employed in the quantification of harmful species in Earth's atmosphere [4], in designing new optical devices and sensors with optimized performances $[5,6]$ and in the fabrication of radiative cooling systems [6]. For such applications, as well as to provide accurate data for numerical simulations, it is necessary to precisely know the complex refractive indices of the used materials. However, precise values are still very scarcely known and experimental set-ups to measure them are not always available, leading to assumptions and conclusions, which may be inappropriate [6].

The complex refractive index $\bar{\eta}$ is a key parameter in the optical description of matter. Note that, in the whole article, complex numbers such as $\bar{\eta}$ will be overlined. $\bar{\eta}$ is defined by Eq. (1).

$\bar{\eta}(v)=n(v)+i \cdot k(v)$

where $v$ is a wavenumber, $n$ is the refractive index and $k$ is the extinction coefficient. $n$ and $k$ characterize the real and imaginary parts of the complex refractive index, respectively. If $k>0$, the medium is absorbent, if $k<0$, the medium is characterized by a gain. Neither $n$ nor $k$ can

Elodie Prud'homme, elodie.prudhomme@insa-lyon.fr | ${ }^{1}$ MATEIS UMR CNRS 5510, Bat. Blaise Pascal, Universit de Lyon, UCBL, 7 Av. Jean Capelle, Villeurbanne, France. ${ }^{2}$ Lyon Institute of Nanotechnologies INL, CNRS UMR 5270, Ecole Centrale de Lyon, Universite de Lyon, 36 Avenue Guy de Collongue, 69134 Ecully Cedex, France. ${ }^{3}$ Lyon Institute of Nanotechnologies INL, CNRS UMR 5270, INSA de Lyon, Universite de Lyon, 7 Avenue Jean Capelle, 69621 Villeurbanne Cedex, France. ${ }^{4}$ MATEIS UMR CNRS 5510, Bat. Blaise Pascal, Universit de Lyon, INSA Lyon, 7 Av. Jean Capelle, Villeurbanne, France. 
be measured directly; they must be determined indirectly from other measurable optical quantities.

All techniques developed to determine complex refractive indices use transmitted or reflected light. In the latter case, the reflectance $R$ is defined by:

$R=\frac{I_{r}}{l_{1}}$

where $I_{r}$ is the intensity of the reflected light and $I_{1}$ the intensity of the incident light.

Among these techniques, infrared ellipsometry is based on the use of transverse linearly polarized light. Magnetic (TM) and electric (TE) transverse linearly polarized lights are obtained with the use of a polarizer. Fresnel reflection coefficients, $\overline{r_{\|}}$and $\overline{r_{\perp}}$, are related to TM and TE polarizations, respectively. As the angle of reflection is equal to the angle of incidence $\theta_{1}$, they are defined in Eqs. (3) and (4) [7].

$\overline{r_{\perp}}=\frac{\overline{\eta_{1}} \cdot \cos \theta_{1}-\overline{\eta_{2}} \cdot \cos \theta_{2}}{\overline{\eta_{1}} \cos \theta_{1}+\overline{\eta_{2}} \cdot \cos \theta_{2}}=\sqrt{R} \cdot \exp ^{-i \delta_{\perp}}$

$\overline{r_{\|}}=\frac{\overline{\eta_{2}} \cdot \cos \theta_{1}-\overline{\eta_{1}} \cos \theta_{2}}{\overline{\eta_{2}} \cdot \cos \theta_{1}+\overline{\eta_{1}} \cos \theta_{2}}=\sqrt{R} \cdot \exp ^{-i \delta_{\|}}$

where $R$ is the measured reflectance and $\delta$ the phase shift of the reflected light with respect to the incident one. As illustrated in Fig. 1, the indices 1 and 2 are defined at the planar interface between two media and are related to the media of incidence and to the analysed material, respectively. Thus, the incident light is characterized by its polarization state and its angle of incidence $\theta_{1} \cdot \theta_{2}$ corresponds to the angle of refraction in the material to be characterized.

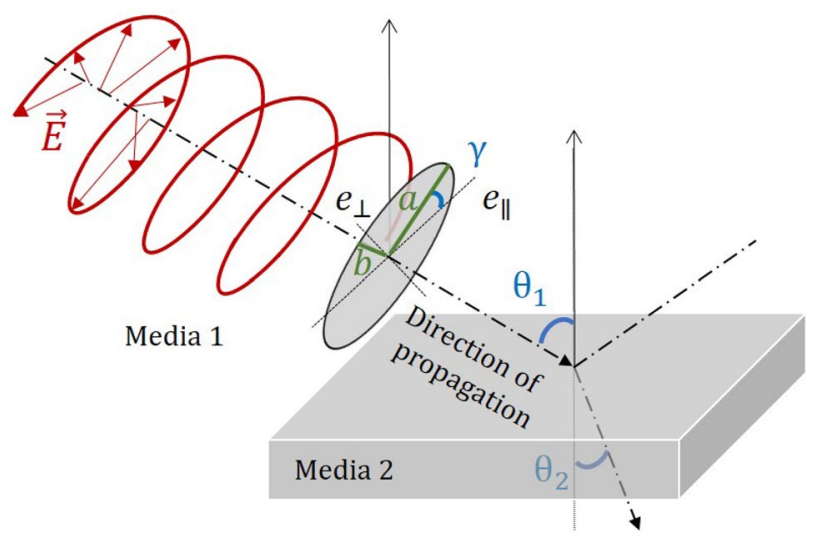

Fig. 1 Evolution of the electric field along the propagation direction and projection of the electric field onto the plane perpendicular to the propagation direction $\left(e_{\|}, e_{\perp}\right)$. The projection is described by an ellipse characterized by parameters $a, b$ and $\gamma$
Using these two states of polarization with a rotational analyser, the phase shift $\delta$ is determined and the complex refractive index can be fully estimated. This method of resolution, known as ellipsometry, is the most used technique because of its robustness and its accuracy. But it is expensive, and therefore it is not available in all laboratories.

Under convergence conditions, the Kramers-Kronig relations link the real and imaginary parts of analytic functions defined in the upper region of Argand plane, i.e., in the half complex plane $\left(\mathbb{R} \times i . \mathbb{R}^{+}\right)$. The phase shift $\delta$ can then be deduced from measurements of reflectance $R$ by Eq. (5) [8].

$\delta(v)=\frac{1}{\pi} p \cdot v \cdot \int_{\mathbb{R}} \frac{\log \sqrt{(R(v))}}{s-v} \mathrm{~d} s=\mathbb{K} \mathbb{K}[\log \sqrt{(R(v))}]$

where p.v. denotes the Cauchy principal values and $\mathbb{K} \mathbb{K}$ the Kramers-Kronig transform. A given angle is then needed to fully determine the complex refractive index, using Eqs. (3) and (4). Hence, with a linear polarizer, any spectrometer can be used to determine $\bar{\eta}$. This option is attractive but suffers from the need of a particular state of polarization. Polarizers do not guarantee a $100 \%$ efficiency and restrict the estimation of the complex refractive index to a limited spectral range. Moreover, a polarizer induces losses and therefore impacts the signal-to-noise ratio. Less expensive and much more common than ellipsometers, the use of a spectrometer alone enables to widen the infrared range over which the complex refractive index can be assessed. This strategy has recently proved to be highly efficient [9] with a near-normal angle of incidence $\left(\theta_{1} \simeq 0\right)$. However, common spectrometers are generally designed with non-zero angles of incidence. To further develop the assessment of complex refractive indices with spectrometers, we have explored the case of non-normal incidence, where a strong dependency on the polarizer efficiency has been observed.

To overcome these limitations, this article reports a new methodology enabling the evaluation of the complex refractive index by a measurement of light reflectance without the use of a polarizer. Section 2 of this article provides details on theoretical backgrounds about the use of a non-normal incidence: first with linearly polarized light, and then with an arbitrary state of polarization. Then, an isotropic material with a low surface roughness has been characterized using a polarizer on the one hand and a classical spectrophotometer to control the value of the incident angle on the other hand. The comparison of the results obtained by these two methodologies provides a proof of concept of the new approach. 


\section{Theoretical framework}

When light-matter interaction is considered to determine the complex refractive index of a material, two media are taken into account. The incident light travels through the first one (air most of the time), with $k_{1}=0$, so $\overline{\eta_{1}}=n_{1}$. Then, the light interacts with the second one, i.e., the material to be characterized, $\overline{\eta_{2}}=n_{2}+i \cdot k_{2}$ (Fig. 1). The two media are separated by a planar interface. The angle between the vector normal to this surface and the direction of propagation of the light is the incident angle $\theta_{1}$. Two theoretical cases will be detailed in this section: when the incident angle is fixed and a polarizer is used (Sect. 2.1), and when the incident angle can be changed and the state of polarization is arbitrary (Sect. 2.2).

\subsection{Given angle of incidence with transverse linearly polarized light}

With TE or TM polarization, $\delta(v)$ is an even function and Eq. (5) becomes Eq. (6) [10].

$\delta(v)=\frac{2 v}{\pi} p \cdot v \cdot \int_{\mathbb{R}^{+}} \frac{\log \sqrt{(R(v))}}{s^{2}-v^{2}} \mathrm{~d} s$

With Eq. (6), the phase shift is discontinues at some singular points on the real and imaginary axes. Therefore, the addition of a corrective term CT to the Kramers-Kronig transform is needed for the evaluation of the phase shift, as in Eq. (7).

$\delta\left(\theta_{1}, v\right)=\mathbb{K} \mathbb{K}[\log \sqrt{R(v)}]+\mathrm{CT}\left(\theta_{1}, M, v\right)$

The phase correction term CT can be estimated in regions of the spectra where $k_{2}=0$, in which case Eq. (7) becomes Eq. (8) [11].

$\left.\delta\right|_{k_{2} \simeq 0}\left(\theta_{1}, v\right)=C T\left(\theta_{1}, M, v\right)$

Thus, CT depends on the incident angle $\theta_{1}$ and on the ratio of the complex refractive indices $M=\frac{\overline{\eta_{1}}}{\bar{\eta}_{2}} \simeq \frac{n_{1}}{n_{2}}[10-13]$.

When the reflectance is measured thanks to specular reflection, the correction term CT is either 0 or $-\pi$, as shown in Table 1. The choice of the value depends on the refractive indices ratio and on the incident angle. To be more precise, it is related to the value of $\theta_{1}$ in comparison with $\theta_{b}$ and $\theta_{c}$ the Brewster's angle and the critical angle $[11,13]$, respectively.
Table 1 Values taken by the phase corrective term $\mathrm{CT}\left(\theta_{1}, M, v\right)$ for TE and TM polarizations in both specular and total attenuated reflections [11-13]

\begin{tabular}{llll}
\hline Polarization & Indices ratio & Angle of incidence & $\mathrm{CT}\left(\theta_{1}, M, v\right)$ \\
\hline$\perp$ & $M>1$ & $/$ & $-\pi$ \\
$\perp$ & $M<1$ & $\theta_{1}<\theta_{c}$ & 0 \\
$\perp$ & $M<1$ & $\theta_{c}<\theta_{1}$ & $g\left(M_{\perp}\right)-\pi^{a b}$ \\
$\|$ & $\theta_{1}<\theta_{b}$ & 0 \\
$\|$ & $M>1$ & $\theta_{b}<\theta_{1}$ & $-\pi$ \\
$\|$ & $M>1$ & $\theta_{1}<\theta_{b}$ & $-\pi$ \\
$\|$ & $M<1$ & $\theta_{b}<\theta_{1}<\theta_{c}$ & 0 \\
$\|$ & $M<1$ & $\theta_{c}<\theta_{1}$ & $g\left(M_{\|}\right)^{c}$ \\
\hline
\end{tabular}

${ }^{\mathrm{a}}: g(M, v)=M(\infty)+\frac{2}{\pi} \arctan \left(\frac{\tau \cdot[M(0)-M(\infty)]}{v}\right),(\tau, M(0), M(\infty)) \in \mathbb{R}^{3}$,

${ }^{\mathrm{b}}: M_{\perp}(v)=2 \arctan \left(\frac{\sqrt{\left(n_{1}(v)^{2} \cdot \sin ^{2} \theta_{1}-n_{2}^{2}(v)\right)}}{n_{1}(v) \cdot \cos \theta_{1}}\right)$

${ }^{c}: M_{\|}(v)=2 \arctan \left(\frac{\sqrt{\left(n_{1}(v)^{2} \cdot \sin ^{2} \theta_{1}-n_{2}^{2}(v)\right)}}{n_{2}^{2}(v) \cdot \cos \theta_{1}} n_{1}(v)\right)$

When attenuated total reflection (ATR) is used, in which case $\theta_{c}<\theta_{1}$, the correction term CT becomes a function of the wavenumber [12] (Table 1). Its evaluation requires to estimate the real part of the refractive index towards $v=0$ and when the wavenumber approaches infinity $v=\infty$ [14]. All these corrections are summarized in Table 1.

Focusing on ATR, high values of reflectance, when $R \rightarrow 1$, can be related to a weak absorptivity $\left(k_{2} \rightarrow 0\right)$. In these conditions, the Fresnel equations lead to the following expression for the phase shift:

$\left.\delta\right|_{k_{2} \simeq 0}(v)= \begin{cases}2 \arctan \frac{\sqrt{n_{1}^{2}(v) \sin ^{2} \theta_{1}-n_{2}^{2}(v)}}{n_{1}(v) \cdot \cos \theta_{1}}, & T M \\ -\pi+2 \arctan \frac{\sqrt{n_{1}^{2}(v) \cdot \sin ^{2} \theta_{1}-n_{2}^{2}(v)}}{n_{2}^{2}(v) \cdot \cos \theta_{1}} n_{1}(v), T E\end{cases}$

As detailed in Table 1, knowing the real part of the refractive index when $k_{2} \rightarrow 0$ is then enough to estimate $g(M, v)$, hence the correction term $\mathrm{CT}\left(\theta_{1}, M, v\right)$ [14]. Thus, no more assumptions are required regarding the value of the real part when $v$ tends towards 0 or towards $\infty$.

The estimation of the value of the complex refractive index in the infrared range covered by the spectrometer is then performed by the following minimization problem [15]: 


$$
\begin{aligned}
& \underset{n_{2}, k_{2}}{\operatorname{minimize}} \\
& \text { subjected to }\left(n_{2}, k_{2}\right) \in\left(\mathbb{R}^{+} \times \mathbb{R}^{+}\right)
\end{aligned} \quad\left\{\begin{array}{l}
\left.\| \sqrt{R(v, \theta)} \cdot \exp ^{-i\left(\mathbb{K} \mathbb{K}(v, \theta)+C T\left(\theta_{1}\right)\right)}-\overline{r_{\|}}\right) \|_{2^{\prime}}^{2}, T M \\
\left.\| \sqrt{R(v, \theta)} \cdot \exp ^{-i\left(\mathbb{K} \mathbb{K}(v, \theta)+C T\left(\theta_{1}\right)\right)}-\overline{r_{\perp}}\right) \|_{2^{\prime}}^{2}, T E
\end{array}\right.
$$

where $\|.\|_{2}^{2}$ is the squared Euclidean norm.

\subsection{Multiple incident angles with arbitrary state of polarization}

A monochromatic wave can be described by a Stokes vector $\mathbf{S}$ [16]:

$\mathbf{S}=\left(\begin{array}{l}I \\ Q \\ U \\ V\end{array}\right)=\left(\begin{array}{c}E_{\|}^{2}+E_{\perp}^{2} \\ E_{\|}^{2}-E_{\perp}^{2} \\ 2 \cdot E_{\|} \cdot E_{\perp} \cdot \cos (\delta) \\ 2 \cdot E_{\|} \cdot E_{\perp} \cdot \sin (\delta)\end{array}\right)$

As illustrated in Fig. 1, in the perpendicular plane to the propagation direction $\left(e_{\|}, e_{\perp}\right), E_{\|}$is the amplitude of the electric field in the direction parallel to the surface of the media 2. $E_{\perp}$ is the amplitude in the direction perpendicular to $E_{\|}$in the plane $\left(e_{\|}, e_{\perp}\right) . \delta$ is the phase shift between the projections onto these two directions. Different intensities and a phase shift, between the parallel and the perpendicular components of the electric field, lead to consider the most general case: the projection of the electromagnetic wave in the plane $\left(e_{\|}, e_{\perp}\right)$ is an ellipse (Fig. 1).

Stokes parameters are related to the parameters describing the ellipse by:

$\mathbf{S}=\left(\begin{array}{l}I \\ Q \\ U \\ V\end{array}\right)=\left(\begin{array}{c}c^{2} \\ c^{2} \cdot \cos 2 \beta \cdot \cos 2 \gamma \\ c^{2} \cdot \cos 2 \beta \cdot \sin 2 \gamma \\ c^{2} \cdot \sin 2 \beta\end{array}\right)$ lights. Following Fresnel equations, the reflection is different from these two states of polarization TE and TM. The associated Stokes vector $\mathbf{S}_{r}$ is then noncollinear to $\mathbf{S}_{1}$ [16]: the ellipse after reflection is different from the ellipse before reflection. The reflectance is then defined by:

$R=\frac{I_{r}}{l_{1}}=\frac{S_{r, 1}}{S_{1,1}}$

where the second index 1 denotes the first element of the vector.

Azzam [17] used this formalism to describe the reflection capacity of an absorbing media with a Mueller matrix. This matrix is named reflection matrix and is denoted $\mathbf{R}$ :

$\mathbf{S}_{r}=\mathbf{R} \cdot \mathbf{S}_{1}$

Nee $[18,19]$ expressed this matrix with Fresnel coefficients only:

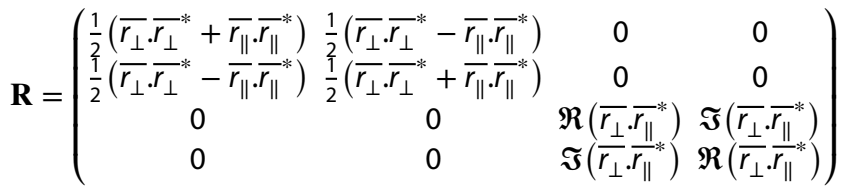

* denotes the complex conjugate. $\mathfrak{R}$ and $\mathfrak{\Im}$ are related to the real and imaginary parts, respectively.

Finally, with the characteristics of the incident light, and with the possibility to measure the reflectance under no less than two different angles of incidence, it is possible to determine completely the complex refractive index. The associated minimization problem is:

$$
\begin{array}{ll}
\underset{n_{2}, k_{2}}{\operatorname{minimize}} & \left\|I_{r}-\left(\frac{R_{11} \cdot S_{1}(1)+R_{12} \cdot S_{1}(2)}{S_{1}(1)}\right)\right\|_{2}^{2} \\
\text { subjected to } & \left(n_{2}, k_{2}\right) \in\left(\mathbb{R}^{+} \times \mathbb{R}^{+}\right)
\end{array}
$$

with $c^{2}=a^{2}+b^{2}$ and $|\tan \beta|=\frac{b}{a}$. $a$ and $b$ are the intensities of the major and of the minor axes of the ellipse, respectively. The orientation of the ellipse is given by the angle $\gamma$ between $e_{\|}$and the major axis, as displayed in Fig. 1.

This description leads to express the incident light $\mathbf{S}_{1}$ as a superposition of shifted transverse linearly polarized where $R_{i j}$ is the $(i, j)^{\text {th }}$ element of the reflection matrix $\mathbf{R}$.

\section{Material and methods}

To illustrate and to compare the two previously described methods, the same sample has been analysed using two experimental configurations: 
Table 2 Vibrational frequencies of silica in the range [750, 2500] $\mathrm{cm}^{-1}[20]$

\begin{tabular}{lll}
\hline Bonding group & Motion & Wavenumber $/ \mathrm{cm}^{-1}$ \\
\hline Si-O & Bend & 813.4957 \\
Si-O & Stretch & 1055.6777 \\
Si-O-Si & Stretch & 1179.5356 \\
\hline
\end{tabular}

- case 1: a standard configuration for which a polarizer is used with the spectrometer. The incident light is polarized and a given incident angle is considered;

- case 2: a device enabling the control of the incident angle is used without any polarizer.

Both approaches lead to solve a minimization problem. Constraints, initialization parameters and methodology of resolution of these problems are detailed in the following section.

\subsection{Material and experimental set-up}

The material is a classical uncoated soda-lime microscopic slide (Thermo Scientific ${ }^{\mathrm{TM}}$ Microscope Slides, Cut, $1 \mathrm{~mm}$, $26 \mathrm{~mm} \times 76 \mathrm{~mm}$ ). In this study, we have focused on the $[750,2500] \mathrm{cm}^{-1}$ wavenumber range that contains few absorption frequencies, as summarized in Table 2. Besides, the presence of a short range order induces a variable broadening of absorbance bands, while there is no long range order due to the amorphous nature of the sample. So, since the optical properties of the glass material analysed in this work are essentially constant above $2500 \mathrm{~cm}^{-1}$, data collected at higher wavenumbers were not required.

Two spectrometers have been used. The first one can be operated with a polarizer, while the second led to better spectral and angular resolutions.

The first apparatus is a commercial VERTEX80 FTIR spectrometer from Bruker coupled to a reflectance accessory to perform angle resolved measurements. A WP25H - Z holographic wire grid polarizer was placed between the light source and the sample. Spectra were collected in the $[400,10,000] \mathrm{cm}^{-1}$ spectral range with a $2 \mathrm{~cm}^{-1}$ spectral resolution using a deuterated triglycine sulfate detector (DTGS). 20 scans were recorded for each acquisition. The wire grid polarizer operates over the $[333,5000] \mathrm{cm}^{-1}$ spectral range with an extinction ratio up to $150: 1$. The transmitted light was polarized perpendicular to the wires (transmission coefficient between 70 and $90 \%$ in the range $\left.[600,5000] \mathrm{cm}^{-1}\right)$. Acquisitions were performed for incident angles of $14^{\circ}, 20^{\circ}, 30^{\circ}$ and $40^{\circ}$. More details about the experimental set-up can be found in the literature [21]. Data collected with this set-up were then numerically reduced to the range $[600,2800] \mathrm{cm}^{-1}$ to both minimize the inaccuracy due to the use of the wire grid and to focus on the spectral range of interest for the glass material.

The second set-up used is the Veemaxll angular device designed by Harrick Scientific with the IS50R Thermo Fisher infrared spectrometer. Spectra have been recorded in the $[650,4000] \mathrm{cm}^{-1}$ spectral range with a $0.4 \mathrm{~cm}^{-1}$ spectral resolution using a DTGS detector. 32 scans were recorded for each acquisition. Acquisitions were performed for incident angles of $35^{\circ}, 40^{\circ}, 45^{\circ}$ and $50^{\circ}$. More details about the experimental set-up can be found in the literature [22]. Data collected with this set-up were then numerically reduced to the range $[745,2500] \mathrm{cm}^{-1}$. To save calculation time, spectra were interpolated in this range reducing from 4388 to 2000 the number of points.

\subsection{Numerical resolution}

Two different strategies for case 1 were investigated. Case 1.1: with the experimental set-up 1, an estimation of the refractive index was performed for each incident angle, using the minimization problem described in (10). Case 1.2: the same spectra have been included together in a unique minimization problem resulting in only one estimation of the refractive index.

The objective function associated to this case was the sums of squares of the objective functions considered in case 1.1. For these two strategies, we used the methodology described in Sect. (2.1) (i.e., linearly polarized light and use of Kramers-Kronig transform). For case 2, with the experimental set-up 2, all spectra obtained for each incident angle have been used in the minimization problem described in (16). For this case, we used the methodology described in Sect. (2.2).

The overall process consisted in the resolution of one minimization problem per wavenumber. Different techniques can be used based on continuous approaches, such as Sequential Quadratic Programming (SQP) [23] or the Interior Point method (IP, potential reduction method) [24], or discontinuous approaches, such as the Genetic Algorithm (GA) [25]. In general, SQP seems to be the fastest. It is therefore the default choice. When optimal solutions to the minimization problem are input values on the boundaries defined by the constraints, IP is preferred. But the presence of local minima is problematic with these two algorithms, as they may not converge towards the optimal solution. The GA algorithm is then considered. As it leads to less precise values, it is used in parallel with a continuous approach. The value of the cost function is used as an arbitrage in the choice of the result [26]. In this paper, the resolution method used is a combination of the different methods discussed above [10]. For each minimization problem, constraints and initializations points had to be 
defined. $n$ and $k$ searched values have been constrained in $\mathbb{R}^{+}$. As the refractive index is continuous in the spectral space, physical sense has been provided to the problem using adequate initialization points, limiting the convergence of nonlinear regressions into a local minimum. The overall process was then solved wavenumber per wavenumber, using the previous $(n, k)$ estimations as guess for the next minimization problem. Each minimization problem was solved twice; the second resolution was initialized with arbitrary values $((n, k)=(3,2))$. The solution with the minimum value of the objective function was chosen. The overall process was solved for the smallest wavenumber first, and then in the ascending order, up to the highest one.

Finally, to evaluate the influence of the acquisition parameters, error bars have been calculated. In case 1.1, the overall process has been performed with values of the incident angle in the range $\Delta \theta_{1} \in[-1,+1]^{\circ}$ (step of $0.2^{\circ}$ ) around the initial value. With 11 cases and 1200 wavenumbers, 52800 minimization problems had to be solved. In case 1.2 , values in the range $\Delta \theta_{1} \in[-1,+1]^{\circ}$ (step of $1^{\circ}$ ) have been considered. This resulted in 81 combinations of incident angular values. 97200 minimization problems have been solved. In case 2 , the overall process has been performed with the following ranges for the parameters of the ellipse: orientation $\gamma_{1} \in[0,90]^{\circ}\left(\right.$ step of $\left.15^{\circ}\right)$ and $b / a$ size ratio in the range $[0.7,0.9]$ (step of 0.1 ). The value of the size ratio was provided by the French research and development department of Thermo Fisher. Finally, 27 combinations have been explored leading to 42,000 minimization problems.

All developments have been coded in MATLAB 2016b, using the interior-point algorithm with the function fmincon for solving minimization problems.

\section{Results}

Case 1.1 has led to four estimations of the complex refractive index, one for each angle of incidence, as shown in Fig. $2 \mathrm{a}-\mathrm{d}$. In case 1.2, all four spectra used in case 1.1 have been used together to obtain a unique estimation (Fig. 2e). In Fig. 2, the spectra of the real and the imaginary parts for these five estimations of the refractive index are plotted together with their error ranges.

The estimations computed using case 1.1 are all similar, except for the range $[950,1250] \mathrm{cm}^{-1}$. In this range, shifts and differences between band intensities are observed. For example, focusing on the real part around $1080 \mathrm{~cm}^{-1}$, the peak maximum is about $n=4.22$ at $1074 \mathrm{~cm}^{-1}$ for $\theta_{1}=14^{\circ}$ and about $n=4.66$ at $1087 \mathrm{~cm}^{-1}$ for $\theta_{1}=40^{\circ}$. Shifts in wavenumber can be explained by the Reststrahlen bands of the $\mathrm{SiO}_{2}$ structure of the material. This particular effect leads to shifts of the associated bands, in reflectance spectra, towards higher wavenumbers when the incident angle increases. The differences in intensities could arise from a bias in calculations, from the use of a polarizer and from the Reststrahlen bands. Kramers-Kronig transforms are defined on infinite space and their use on a finite range generally leads to biased values of the phase shift, and so, of the refractive index too. Moreover, polarizers do not provide a 100\% TM light: indeed, the polarization state is different along the spectral range. Consequently, the uncertainty on the phase shift is increased. In case 1.1 confidence intervals are tight: from $10^{-3}$ for $\theta_{1}=15^{\circ}$ to $10^{-2}$ for $\theta_{1}=40^{\circ}$, as shown in Fig. 2 (iii). This increase is caused by the evolution of reflectance when the incident angle increased $[11,13]$. In case 1.2 , the confidence intervals are about the same order of magnitude as in case 1.1 and mean values lie between the mean values found for case 1.1. The first methodology of resolution is then robust against the angular dependencies but is strongly influenced by Reststrahlen bands.

The same sample was analysed in cases 1.1 and 2, leading to similar estimations in both cases. In Fig. 3, they are
Fig. 2 Spectra of (i) $n$ and (ii) $k$ for $\mathbf{a} \theta_{1}=40^{\circ}, \mathbf{b} \theta_{1}=30^{\circ}, \mathbf{c}$ $\theta_{1}=20^{\circ} \mathbf{d} \theta_{1}=14^{\circ}$ for case 1.1 , and e for case 1.2. (iii) Zoom for the real part in the range $[1070,1095] \mathrm{cm}^{-1}$ (see square frame in (i)). For all cases, mean values are plotted in dotted lines and $95 \%$ confidence intervals are represented in solid coloured lines
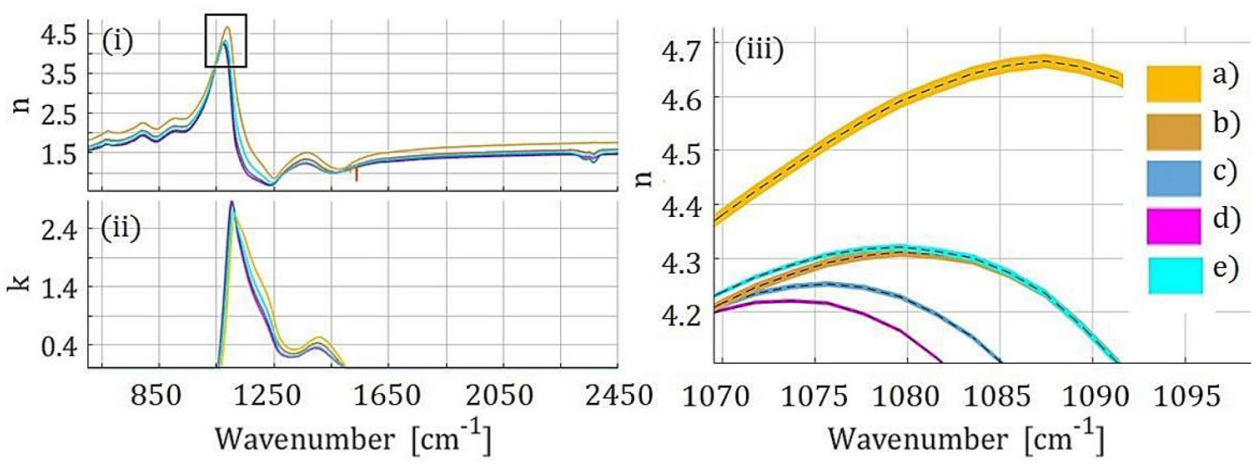
Fig. 3 (i) Mean values for real part of the refractive index for a case 1.1 related to $\theta=40^{\circ}, \mathbf{b}$ case 2 and c Palik's value [27]. (ii) $95 \%$ confidence intervals in coloured solid lines and mean values in dotted lines. (iii) Mean values for the imaginary part

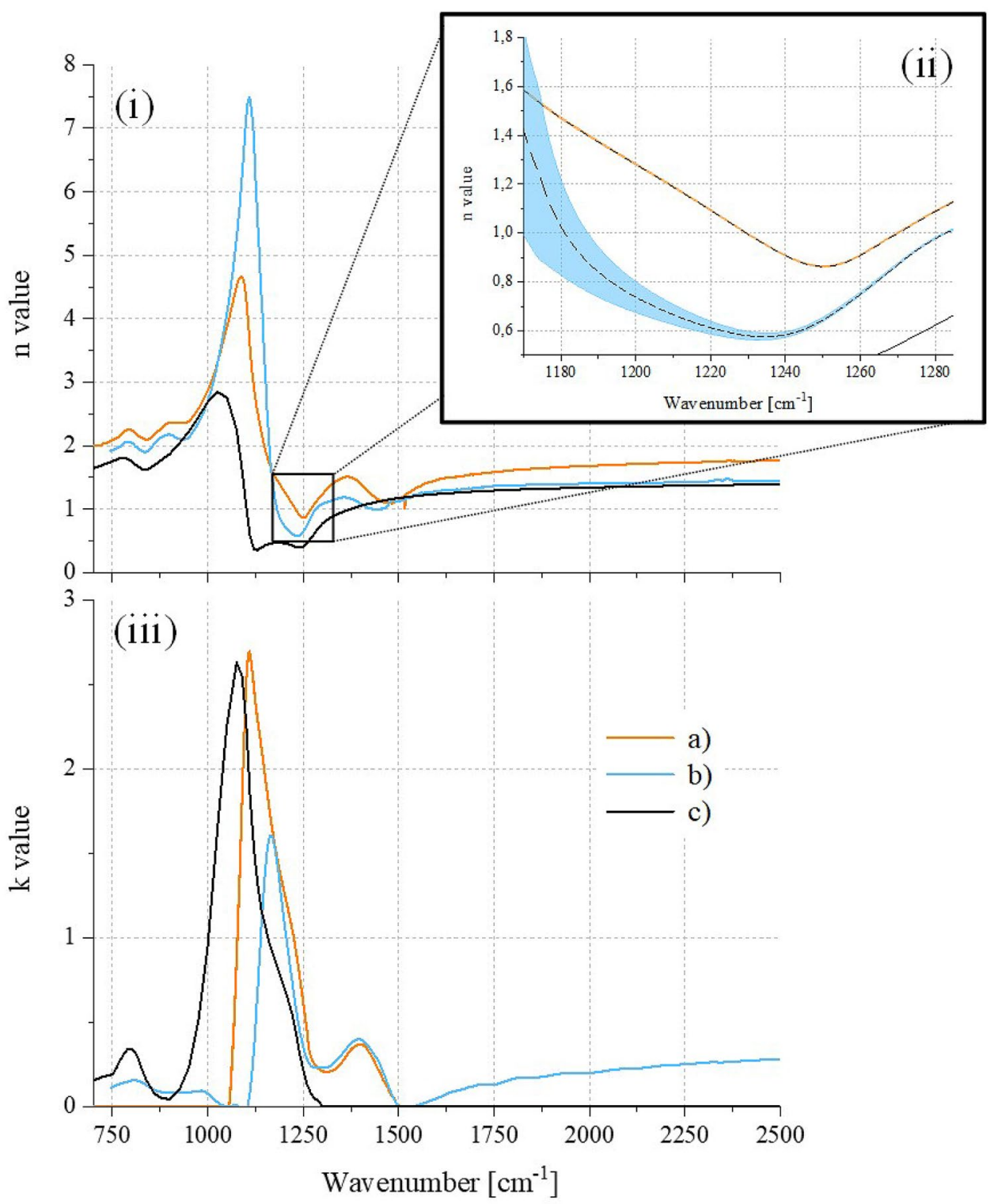

compared to the complex refractive index of amorphous silica from Palik [27]. They are both in agreement with Palik's values, but a shift of the main band is observed in the range $[1000,1250] \mathrm{cm}^{-1}$. As explained before, shifts in this range are expected due to the Reststrahlen band of the $\mathrm{SiO}_{2}$ structure as in [9]. Moreover, the estimation obtained in case 2 took into account incident angles between 35 and $50^{\circ}$. The greater shift towards higher wavenumbers for case 2 (Fig. 3 (ii)) compared to Palik's values is in accordance with these expectations. The main differences between Palik's [27] values and estimations from both methodologies may be explained by two reasons. First of all, the composition of the glasses characterized in this work is not pure amorphous silica as for Palik. Thus, additional bands are observed. Secondly, the sensitivity of the two detectors used in both set-ups decreases below
$850 \mathrm{~cm}^{-1}$. Hence, as observed in Fig. 3 (i) and (iii), the values of reflectance below this wavenumber are underevaluated by the detectors, and thus the absorptivity are underestimated. For example, a closer look at the results obtained by case 2 (Fig. 3 (iii)) shows a shoulder at $800 \mathrm{~cm}^{-1}$, which intensity is significantly lower than Palik's estimation. The literature already mentions these differences according to the glass slides used $[28,29]$.

Focusing on the second approach, the bandwidth of the error range lies between 0.1 and 0.4 , as plotted in Fig. 3 (ii). For instance at $1107 \mathrm{~cm}^{-1}$, mean value of the real part is $n=6.81$ with $\gamma=90^{\circ}$ and is $n=8.17$ with $\gamma=0^{\circ}$. The fact that the results obtained by this methodology are spread out over a large range of values is due to the uncertainty on the state of polarization of the incident light. 
Finally, the contradictory requirements of the experimental possibilities and the numerical needs penalize the case 1.1 (a given incident angle and a transverse polarization): the calculus needs a wide spectral range while the polarizer is efficient only on a restricted spectral range. However, a given angle of incidence is needed, and without Reststrahlen band this method is a very simple and practical way to obtain a first estimation of the refractive index. On the contrary, leading to more accurate results, the second methodology, developed in this work (multiple incident angles without any specific polarization), has proven all its power dealing with arbitrary states of polarization.

\section{Conclusions}

A new theoretical framework has been explored and applied leading to estimations of the complex refractive index of an isotropic material from a measurement of the reflected light with any state of polarization. In practice, any spectrometer can be used to achieve this objective, and no information on the polarization state of the light is required.

For a given incident angle, the use of a polarizer and a numerical resolution including Kramers-Kronig transforms is still the only way to proceed. It has been shown how to correct these transforms when collection of spectra can be performed with multiple incident angles. Limitations induced by the use of a polarizer have also been pointed out.

Furthermore, a new theoretical background developed in this work enables estimating the complex refractive index of a material with equivalent accuracy as when obtained from ellipsometry measurements. To do so, efforts on the evaluation of the state of polarization should be made. Finally, using common spectrometers to obtain a first approximation of the complex refractive index could permit to save money, and moreover, to democratize the assessment of such an important parameter.

Acknowledgements We would like to thank R. Pujol and B. Beccard for fruitful discussions.

Author Contributions $\mathrm{TB}, \mathrm{AB}$ and $\mathrm{RO}$ were involved in methodology and formal analysis; TB, EP, ST and GF contributed to investigation; $T B$ and EP contributed to visualization; TB, AB, RO, EP, ST and GF contributed to writing - original draft preparation. All authors have read and agreed to the published version of the manuscript.

Funding This work was supported by the French Ministry of Higher Education and Research and ENS Paris-Saclay.

\section{Compliance with ethical standards}

Conflict of interest The authors declare that they have no conflict of interest.

\section{References}

1. Petibois C, Déléris G (2006) Chemical mapping of tumor progression by FT-IR imaging: towards molecular histopathology. Trends Biotechnol 24(10):455-462

2. Grigorev R, Kuzikova A, Demchenko P, Senyuk A, Svechkova A, Khamid A, Zakharenko A, Khodzitskiy M (2020) Investigation of fresh gastric normal and cancer tissues using terahertz timedomain spectroscopy. Materials 85(13):85

3. Sotiropoulou S, Papliaka ZE, Vaccari L (2016) Micro FTIR imaging for the investigation of deteriorated organic binders in wall painting stratigraphies of different techniques and periods. Microchem J 124:559-567

4. Mahieu E, Chipperfield MP, Notholt J, Reddmann T, Anderson J, Bernath PF, Blumenstock T, Coffey MT, Dhomse SS, Feng W, Franco B, Froidevaux L, Griffith DWT, Hannigan JW, Hase F, Hossaini R, Jones NB, Morino I, Murata I, Nakajima H, Palm M, Paton-Walsh C, III JMR, Schneider M, Servais C, Smale D, Walker KA (2014) Recent Northern Hemisphere stratospheric $\mathrm{HCl}$ increase due to atmospheric circulation changes. Nature 515(7525):104-107

5. Marques Lameirinhas R, Torres J, Baptista A (2020) The influence of structure parameters on nanoantennas optical response. Chemosensors 42(8):85

6. Zhang X, Qiu J, Zhao J, Li X, Liu L (2020) Complex refractive indices measurements of polymers in infrared bands. J Quant Spectrosc Radiat Transf 252:107063

7. Liu J-M (2005) 1: background. In: Liu J-M (ed) Photonic devices. Cambridge University Press, Cambridge, pp 1-68

8. Lucarini V, Peiponen K-E, Saarinen JJ, Vartiainen EM (2005) Kramers-Kronig relations in optical materials research, Springer series in optical, sciences edn. Springer, Berlin

9. Kelly-Gorham MR, Devetter BM, Brauer CS, Cannon BD, Burton SD, Bliss M, Johnson TJ, Myers TL (2017) Complex refractive index measurements for $\mathrm{BaF} 2$ and $\mathrm{CaF} 2$ via single-angle infrared reflectance spectroscopy. Opt Mater 72:743-748

10. Bonnal T (2018) Chapter 4, in Dveloppements de modles optiques et de mthodes non supervises de rsolution des problmes bilinaires : application limagerie vibrationnelle. PhD Thesis, Lyon University

11. Yamamoto K, Masui A, Ishida H (1994) Kramers-Kronig analysis of infrared reflection spectra with perpendicular polarization. Appl Opt 33(27):6285-93

12. Plaskett JS, Schatz PN (1963) On the robinson and price (Kramers-Kronig) method of interpreting reflection data taken through a transparent window. J Chem Phys 38(3):612-617

13. Yamamoto $K$, Ishida $H$ (1994) Kramers-Kronig analysis of infrared reflection spectra with parallel polarization for isotropic materials. Spectrochim Acta A 50(12):2079-2090

14. Bardwell JA, Dignam MJ (1985) Extensions of the Kramers-Kronig transformation that cover a wide range of practical spectroscopic applications. J Chem Phys 83(11):5468-5478

15. Kuzmenko $A B$ (2005) Kramers-Kronig constrained variational analysis of optical spectra. Rev Sci Instrum 76(8):83108

16. Bohren CF, Huffman DR (1983) Absorption and scattering of light by small particles. Wiley, New York

17. Azzam RMA (1978) Propagation of partially polarized light through anisotropic media with or without depolarization: a differential 4x4 matrix calculus. J Opt Soc Am 68(12):1756 
18. Nee SMF (1996) Polarization of specular reflection and near-specular scattering by a rough surface. Appl Opt 35(19):3570

19. Nee S-MF (2002) Principal Mueller matrix of reflection and scattering measured for a one-dimensional rough surface. Opt Eng 41(5):994

20. Tsu DV, Lucovsky G, Mantini MJ (1986) Local atomic structure in thin films of silicon nitride and silicon diimide produced by remote plasma-enhanced chemical-vapor deposition. Phys Rev B 33:7069-7076

21. Humlicek J (2005) 1: polarized light and ellipsometry. In:Tompkins HG, Irene EA (eds) Handbook of ellipsometry. William Andrew Publishing, Norwich, NY, pp 3-91

22. Bonnal T, Prudhomme E, Foray G, Tadier S (2019) Infrared mapping of inorganic materials: a supervised method to select relevant spectra. Chemom Intell Lab Syst 188:14-23

23. Powell MJD (1978) A fast algorithm for nonlinearly constrained optimization calculations. In: Watson GA (ed) Numerical analysis. Springer, Berlin Heidelberg, pp 144-157

24. Wright MH (1992) Interior methods for constrained optimization. Acta Numer 1:341-407
25. Lucasius C, Kateman G (1993) Understanding and using genetic algorithms part 1. Concepts, properties and context. Chemom Intell Lab Syst 19(1):1-33

26. Bonnal T (2018) Chapter 2, In Dveloppements de modles optiques et demthodes non supervises de rsolution des problmes bilinaires :application limagerie vibrationnelle PhD Thesis, Lyon University

27. Palik ED (1991) Handbook of optical constants, vol. 2

28. Luo J, Smith NJ, Pantano CG, Kim SH (2018) Complex refractive index of silica, silicate, borosilicate, and boroaluminosilicate glasses analysis of glass network vibration modes with specularreflection IR spectroscopy. J Non-Cryst Solids 494:94-103

29. Rubin M (1985) Optical properties of soda lime silica glasses. Sol Energy Mater 12(4):275-288

Publisher's Note Springer Nature remains neutral with regard to jurisdictional claims in published maps and institutional affiliations. 